\title{
Monitoring the thermal deformation of steel beams via vision metrology
}

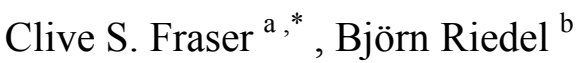 \\ ${ }^{a}$ Department of Geomatics, University of Melbourne \\ Melbourne, Victoria 3010 Australia \\ ${ }^{\mathrm{b}}$ Institute of Geodesy \& Photogrammetry, Technical University of Braunschweig \\ D-38106 Braunschweig, Germany
}

\begin{abstract}
The multi-epoch deformation monitoring of a series of super-hot steel beams by digital closerange photogrammetry is reported. An on-line configuration of three CCD cameras was established to measure both stable reference points and targets subject to positional displacement. Measurements for each beam were conducted at 70-80 epochs over two hours as the steel cooled from $1100 \mathrm{C}$ to near room temperature. Special targeting was required to accommodate the changing colour of the beams from white-hot to brown as they cooled and ensure target survival through a large temperature range. A computational approach was employed whereby the photogrammetric triangulation process for any given recording epoch utilised all images obtained up until that time. The paper discusses all aspects of the project in which seven beams were monitored to a dimensional tolerance of close to $1 \mathrm{~mm}$ (RMS 1sigma).
\end{abstract}

Key Words: vision metrology; deformation measurement; multi-epoch data processing; automated image measurement.

\section{Introduction}

Accompanying recent advances in close-range digital photogrammetry for industrial measurement, there have been broader applications of automated vision metrology techniques for deformation monitoring. Reported projects have included the determination of deformation of industrial tooling (Beyer et al., 1995; Fraser, 1996), large electric motors (Fraser, 1999) and formed concrete sections (Niederöst and Maas, 1997). This paper deals with the monitoring of deformation of steel beams. Shown in Fig. 1 is a $9 \mathrm{~m}$ steel beam positioned within a thermal testing facility at the Technical University of Braunschweig, Germany. As part of a material testing process, there was a requirement to measure the thermal deformation of a number of such beams, each of a different cross section and different steel composition. Finite element models had been formulated covering the deformation of each beam type over a temperature range of $1200 \mathrm{C}$ to $0 \mathrm{C}$, and it was desired to evaluate the integrity of these models. This could only be achieved through a direct measurement of surface point deflections across a wide temperature range, the range being from close to $1100 \mathrm{C}$ to normal room temperature.

\footnotetext{
* Corresponding author. E-mail: c.fraser@unimelb.edu.au (C.S. Fraser).
} 
What would otherwise have constituted a reasonably straightforward deformation measurement task became a potentially very difficult operation due to the very high temperatures involved. Contact measurements were out of the question as were non-contact optically-based 3D co-ordinate determination methods that could not sustain a measurement rate of 1-2 points per second. This was because of an expressed desire to monitor the beams over an approximately two-hour period as they cooled from $1100 \mathrm{C}$ to about $50 \mathrm{C}$, initially with an epoch of measurement occurring every 15 seconds. After a review of candidate 3D measurement technologies it became clear that a photogrammetric approach would be the most viable, though it would not be free of practical difficulties. The use of digital theodolite systems was precluded both because all points needed to be measured simultaneously at a given epoch, and the high-temperature environment was not conducive to theodolite operation. The latter reason also accounted for why contact measurements could not be considered, and targetless ranging systems could not meet the accuracy requirements.

This paper describes the successful conduct of the project in which close-range digital photogrammetry was employed for the deformation monitoring of seven steel beams similar to that shown in Fig. 1. Some 70-80 epochs of positional data were recorded for each beam, with point deflections being determined at each of the targeted positions indicated in the figure. Given the volume of data to be recorded it was necessary to design a highlyautomated, on-line data processing system, which had to incorporate special features to overcome problems associated with the high-temperature environment. One such problem, for example, was that once the beam was uncovered and commenced cooling, its colour changed from a bright yellow (so-called white-hot) through orange and red, and finally to brown. This posed problems in selecting a target that would maintain a high contrast against the changing colour and brightness of the hot steel. Moreover, it was no straightforward matter to find a signalisation material that would withstand such high temperatures.

In the following sections, the deformation measurement requirements are presented in more detail and the design of the photogrammetric measurement system is described. A noteworthy feature of the system is the level of automation employed and the strategy for online, multi-epoch photogrammetric orientation/triangulation. Finally, results of the project are summarised.

\section{Photogrammetric network}

\subsection{Physical constraints}

As indicated in Fig. 1, the photogrammetric network needed to accommodate two categories of target. The first comprised the 10-15 monitoring points on each beam, whereas the second included about 30 targets upon the stable structure behind the thermal testing pit. Given that the beam had to be uncovered prior to a sequence of measurements, and considering the temperatures involved, it was not practical to position monitoring points on the far wall of the pit. Hence, the less than optimal distribution of targets shown in Figs. 1 and 2. The stable points served two purposes. The first was to provide a fixed reference system with respect to which epoch-to-epoch point displacements would be determined, and the second was to provide the necessary broad spread of monitoring points to support photogrammetric bundle adjustment. The adopted imaging configuration had to take into account the physical constraints imposed due to the high-temperature of the steel. 


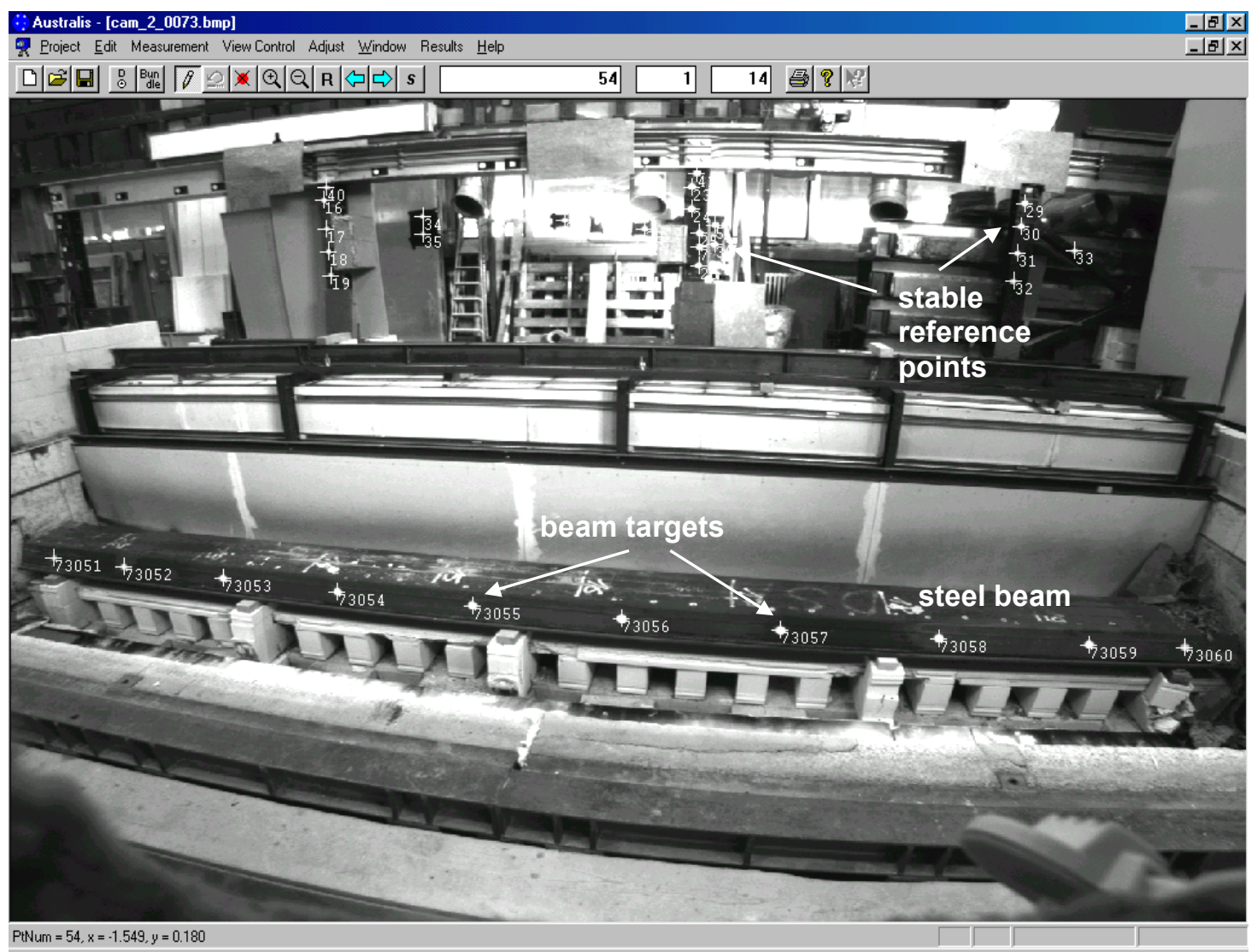

Fig. 1. Targeted steel beam mounted within the thermal test facility, as viewed from the centre camera station.

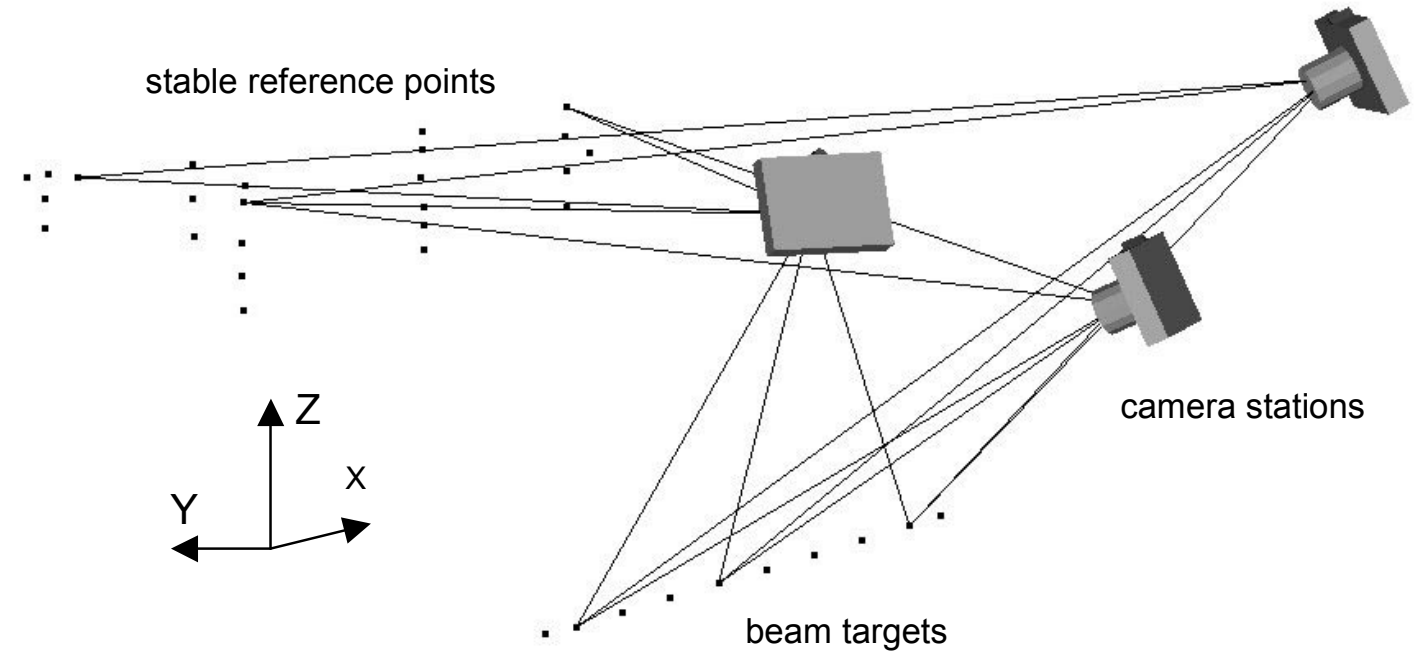

Fig. 2. Photogrammetric network comprising 3 camera stations and 40 object points, 30 stable targets and 10 subject to displacement. 


\subsection{Imaging configuration}

A three-camera configuration was employed for the photogrammetric network, which for any epoch comprised all targets on the steel beam along with 25 or so of the stable, background targets. Fig. 2 illustrates the basic network geometry. The digital cameras selected were Jai, model M1 progressive scan cameras with imaging arrays of $1300 \times 1030$ pixels and a pixel size of $6.7 \mu \mathrm{m}$ square. The two outer cameras were fitted with $8 \mathrm{~mm}$ lenses, which provided a horizontal field of view of $57^{\circ}$, whereas the centre camera had an $85^{\circ}$ field of view from its $4.8 \mathrm{~mm}$ lens. The wider field of view at the centre camera was necessary due to limitations in the available set-back distance for this imaging station. The average camera-to-object distance for the outer two cameras was $9.6 \mathrm{~m}$ (imaging scale of 1:1200), whereas that for the centre camera was $6.7 \mathrm{~m}(1: 1400$ scale).

Fig. 3 shows one of the Jai CCD cameras mounted on its supporting structure. With the 3station network indicated in Fig. 2, coupled with an image co-ordinate measurement accuracy via intensity weighted centroiding of 0.1 pixel, triangulation precision (RMS 1-sigma) of close to $0.5 \mathrm{~mm}$ could be anticipated for points along the beam. At each measuring epoch, the three images were acquired sequentially, and then processed via a single frame-grabber board and stored within the host PC in a total time of 0.2 seconds. It was initially intended that the processing of the triangulation of the targets would be on-line. The multi-epoch photogrammetric restitution approach to be adopted had therefore to fully support this feature.

\subsection{Targeting}

In contrast to the standard targeting used for the stable reference points, the photogrammetric targets which constituted deformation monitoring points on each beam had to display two essential characteristics. Firstly, the material had to survive temperatures exceeding $1100 \mathrm{C}$, and secondly the target had to maintain its contrast against a background that was dramatically changing in colour over time. The targeting material selected was a highly heatresistant ceramic $\left(\mathrm{Al}_{2} \mathrm{O}_{3}\right)$, which maintained its off-white colouring over the full range of temperatures. Custom-made circular targets of $6 \mathrm{~cm}$ diameter were fixed to the beam with stable, steel bolts, as indicated in Fig. 1. The average diameter of both beam and stable targets within the imagery was 6-7 pixels.

This signalisation strategy had only one drawback, namely that to survive intact the targets had to be about $5 \mathrm{~mm}$ thick, which meant that there would be a bias in image centroid determination whenever the incidence angle was oblique to the surface plane (e.g. Dold, 1996). There was no ready solution for this shortcoming, though there was the consolation that all epochs would be similarly effected and thus a degradation in the measurement of beam deflection was not anticipated. A degradation in the triangulation closures was anticipated, however, and an effective image co-ordinate measuring accuracy of closer to 0.2 pixel was predicted.

Preliminary tests were conducted to ensure that as a beam cooled and changed colour the centroid of the ceramic 'dot' target remained invariant. At temperatures below about $800 \mathrm{C}$ there was no difficulty validating this assumption. At higher temperatures, however, particular care had to be paid to selecting an appropriate threshold greyscale value to separate the target and background. It turned out that this normally automatic process would require manual monitoring and a degree of operator intervention within the measurement at the first (hottest) dozen or so epochs. 


\section{Data processing}

For each steel beam, $70-80$ epochs of measurement were to be performed, with the full test sequence consuming between 90 minutes and two hours. The first 40 measured epochs were required at 15 -second intervals, after which the frequency was reduced to a measurement every minute for 20 minutes. The final $10-25$ epochs were recorded at 5-minute intervals. To support the large volume of photogrammetric processing, and to meet the desire for online monitoring, the data processing scheme indicated in Fig. 4 was adopted. This had two essential components, a preliminary off-line phase and an on-line photogrammetric triangulation process.

\subsection{Sensor calibration and initial exterior orientation}

The three-camera configuration did not support the prospect of sensor self-calibration due to the small number of camera stations and each camera was therefore pre-calibrated off-site via self-calibrating bundle adjustments employing 12-camera stations and 40 object points. This preliminary process also enabled a verification of the metric assumptions made for the Jai CCD cameras, namely that triangulation closures of better than 0.05 pixel would be attainable. Such a goal was realised, though it must be recalled that optimal calibration was not a prerequisite for this application since absolute positioning accuracy was of secondary importance compared to the precise determination of relative point movements. Thus, so long as any small systematic errors in sensor calibration or exterior orientation were constant within a beam measurement sequence, the resulting bias in triangulation at each epoch could also be assumed to be near constant. The maximum point movement, after all, was only about $5 \mathrm{~cm}$ (in length), which at image scale represented a variation in centroid position of only 6 pixels or less over the entire 70 epochs.

Following the camera calibration process, which was conducted only once, a preliminary exterior orientation/triangulation accompanied the set-up phase for each beam. With the cameras in place, imagery was recorded of the background points, and also, when possible, of points on the beam prior to its covering for heating. The subsequent bundle adjustment established the preliminary exterior orientation for the network, along with initial triangulated co-ordinates for points on the beam.

\subsection{On-line processing}

The main aim of on-line data processing was the determination at each measurement epoch of the deformation parameters associated with the current temperature. Thus, a system was designed, whereby a photogrammetric triangulation would be performed as the imagery was acquired. This could also apply to the preliminary exterior orientation described above. The distinctive feature of the on-line operation was that rather than performing a bundle adjustment with the latest three images only, the adjusted network would comprise all image co-ordinate observations recorded up until that point, as indicated in Fig. 4. Thus, if there were 30 stable background points and 10 targets on the beam for each epoch, the bundle adjustment at Epoch 50 would comprise 150 images and 530 object points. The stable points would be common to all epochs whereas the targets on the beam would be re-labelled and thus constitute 'new' object points at each epoch. 


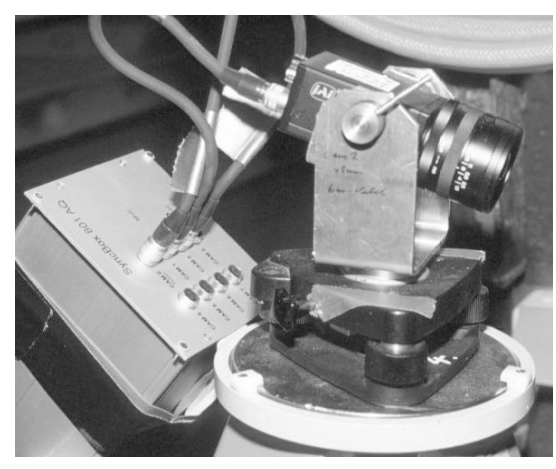

Fig. 3. One of the $1.3 \mathrm{~K} \times 1 \mathrm{~K}$ Jai CCD camera mounted in position.

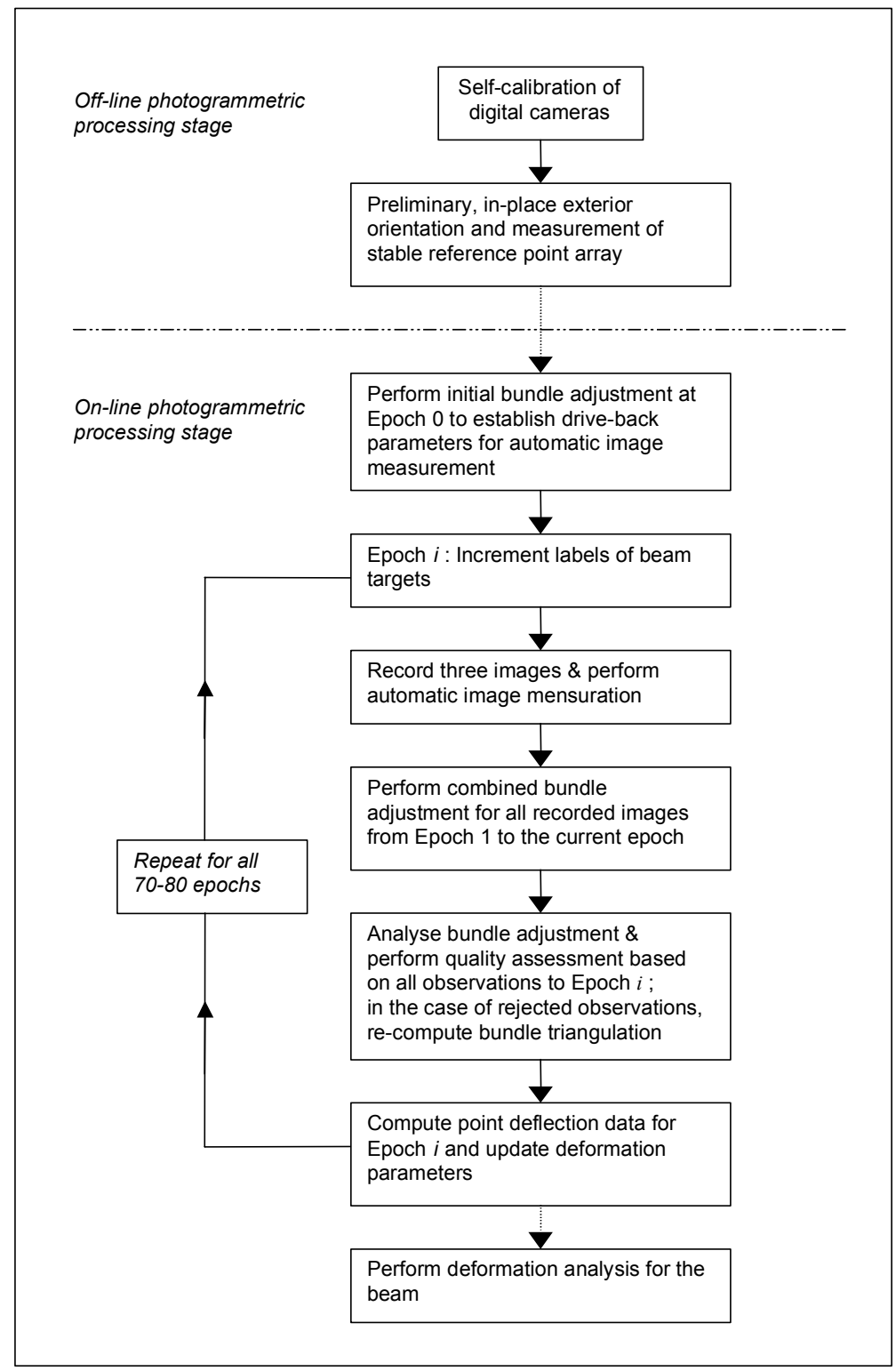

Fig. 4. Computational sequence of the off-line and on-line measurement processes. 
From a photogrammetric standpoint the use of all available data within the bundle triangulation at each epoch was very desirable. Firstly, the substantially increased redundancy enhanced both network precision and reliability, and thus the detection of observational errors, at least for the stable point field. Secondly, and arguably of more practical significance, was the fact that a reference co-ordinate system common to all epochs was implicitly defined via this approach. Although subsequent adjustments could lead to small variations in triangulated object point co-ordinates for previous epochs, at the final epoch all object points would belong to a uniform datum, this being nominally defined in terms of the pre-surveyed XYZ co-ordinates of some of the stable reference points. Provision of a common datum simplified the computation of deformation parameters. All bundle adjustments were minimally constrained, free-network solutions implemented via inner constraints (e.g. Fraser, 1984).

The on-line photogrammetric processing system was realised through a modification of the Australis system for off-line digital close-range photogrammetry (Fraser and Edmundson, 2000). Extra features were added to this software system to support the cumulative, multiepoch processing, a critical component of which was the automatic measurement of all image points. This was a relatively straightforward process since the images from the previous epoch could be employed to 'drive' the measuring process at the current epoch.

In spite of the endeavours undertaken to ensure fast on-line image measurement and photogrammetric triangulation, the requirement for the provision of instantaneous results at each epoch was not actually called upon for the seven beam measurements conducted. Instead, all images were stored for later processing, which was nevertheless conducted as originally intended. This delayed processing did, however, allow a further option to be added to the Australis processing system, namely the ability to choose any subset of epochs to process, in any desired order. In the beam measurements conducted it was found that manual intervention in the grey value thresholding of the ceramic targets was only required for the first 10-15 epochs when the temperature was still above 800C. To locate such image points it was easier to run the processing backwards, commencing at the last epoch and proceeding towards the first. Shown in Fig. 5 are six images of the same targeted section of one of the beams. In the first image (a), which corresponds to the time the beam is uncovered, it is difficult to accurately measure target centroids of the near-white targets against the white-hot background. After 4-5 minutes, as the beam darkens, the measurement process commences. The remaining images in the figure, (b) - (f), show the same three targets at Epochs 1, 5, 10, 20 and 40. These illustrate why an improvement in target centroiding could be expected to accompany the change in colour of the steel from a radiant yellow through to a dull brown.

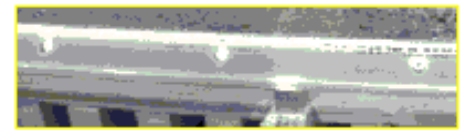

(a)

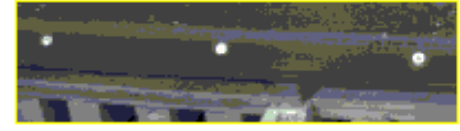

(d)

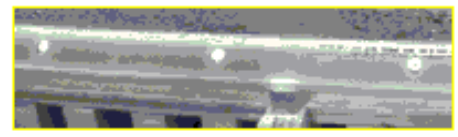

(b)

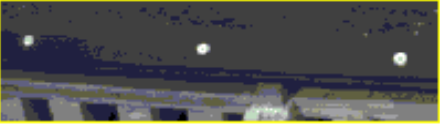

(e)

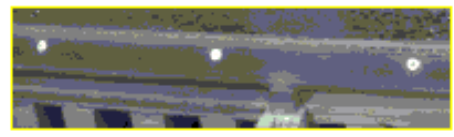

(c)

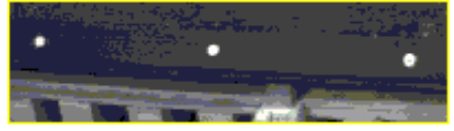

(f)

Fig. 5. The impact of changing temperature on target contrast: the same three targets are shown for the instant the beam is uncovered (a) and for Epochs 1, 5, 10, 20 \& 40 (b-f, respectively). 


\section{Results}

Following on from the experimental design and system development phase, seven beam measurements were conducted, with each following the operational procedure described above. The primary initial outcome of these surveys was the determination of the pattern of surface deformation exhibited by each steel beam as it underwent a change of temperature of close to 1100 C. Shown in Figs. 6 and 7 are plots of position versus time for the targets on the beam, for the two co-ordinate axes $\mathrm{X}$ (longitudinal axis) and $\mathrm{Z}$ (vertical). The maximum point displacement in both cases was about $5 \mathrm{~cm}$, whereas the deformation in the lateral direction (Y) was considerably less at under $2 \mathrm{~cm}$. The measured point displacement data was delivered to the structural engineers for further proprietary analysis. Noteworthy in the figures is the time lag from the commencement of the imaging sequence to the first measured epoch after $1200 \mathrm{~s}$, since at the beginning steel and targets were indistinguishable.

While in most respects the individual photogrammetric measurements were performed according to design, the image mensuration accuracy, and therefore the subsequent precision of triangulation, was less than was anticipated at the network design stage. As is indicated in Fig. 5, the poor contrast between the targets and the hot, light-coloured steel degraded the precision of the weighted centroiding operation.

Moreover, with the cameras having variable gain, the changing radiance of the hot steel also led in the early epochs to a measurable degradation of precision in the automatic centroid determination for a number of the stable background targets, which became a little underexposed. The apparent jumps in point deformation that are noticed in some of the first measuring epochs in Figs. 6 and 7 are likely attributable to these radiometric effects, for both stable targets and those on the beam. Notwithstanding these shortcomings, acceptable results were obtained with the final representative precision (RMS 1-sigma) for triangulated object point co-ordinates being close to $1.0 \mathrm{~mm}$ for the monitoring targets on the beam.

The observational residuals within the imagery displayed a significant systematic error component due to the above mentioned target thickness, which could be anticipated to be near constant over a series of epochs. It was thus expected that the relative accuracy of measured deformations would be superior to the precision indicated by the variances associated with the photogrammetric triangulation. There was no practical means to rigorously verify this assumption, though the smoothness of the resulting curves of point displacements versus time supported the conclusion that sub-millimetre point displacement accuracies were being achieved. As it happened, the final RMS value of image co-ordinate residuals in the approximately 210 -station, 800 object point bundle adjustments for the 70 epochs of data averaged $1.6 \mu \mathrm{m}$, or close to 0.2 pixels. In terms of relative positioning accuracy, monitoring points on each beam were positioned to only about 1: 9000 of the size of the object, which is modest by modern vision metrology standards, though not unexpected for the small-format Jai CCD cameras and the physical conditions involved, which included the matter of less than optimal measurement of image point centroids referred to earlier.

\section{Concluding remarks}

The successful multi-epoch deformation measurement of the seven steel beams offers a further example of how vision metrology can be applied in difficult environments where the application of alternative 3D co-ordinate measurement technologies is precluded. In this instance, standard off-the-shelf CCD imaging sensors were configured in a three-station, on- 
line photogrammetric network which could yield, automatically, deformation data at the time of each of the 70 - 80 monitoring epochs. In order to support the automated image mensuration and data processing, the Australis software system for off-line digital close-range photogrammetry was modified to support on-line operation. A central feature of the photogrammetric data reduction was the ability to incorporate all observational information within the bundle adjustment at each new epoch of recording.

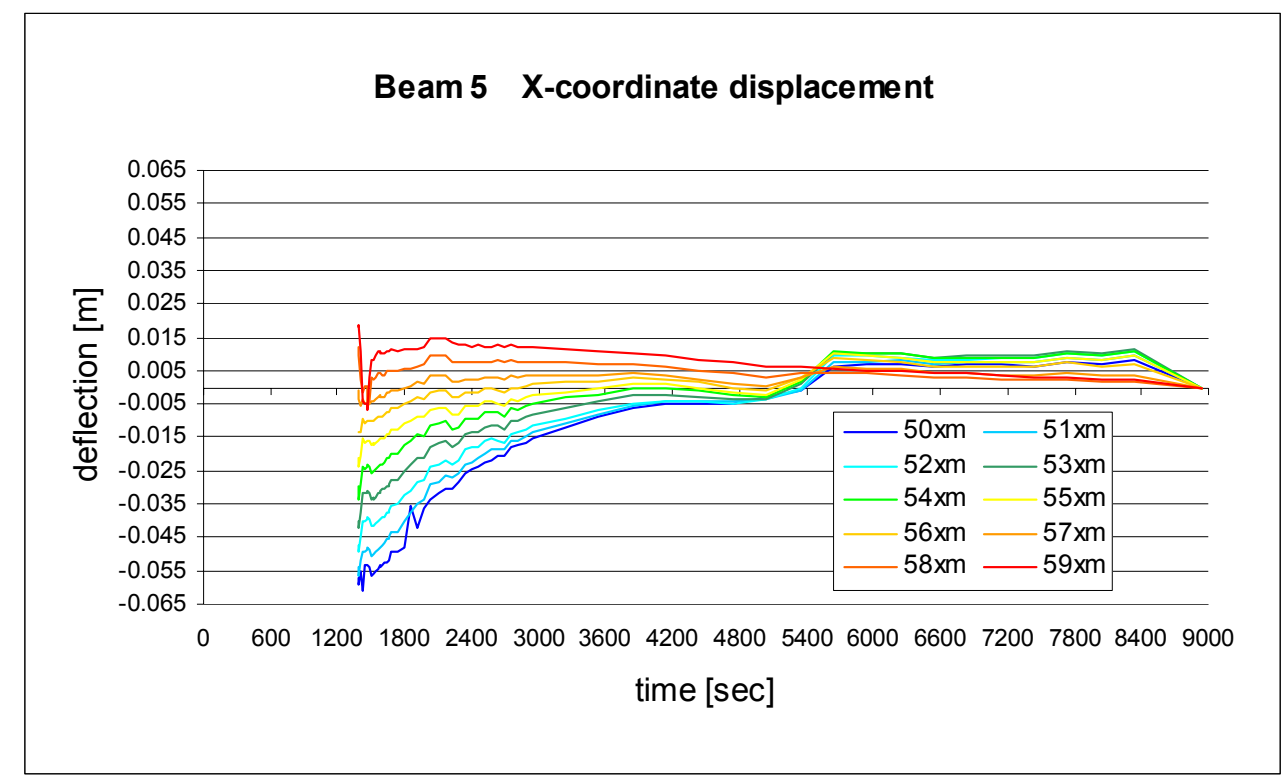

Fig. 6. Plots of positional displacement versus time for the 10 targets on Beam 5, for the longitudinal axis (X).

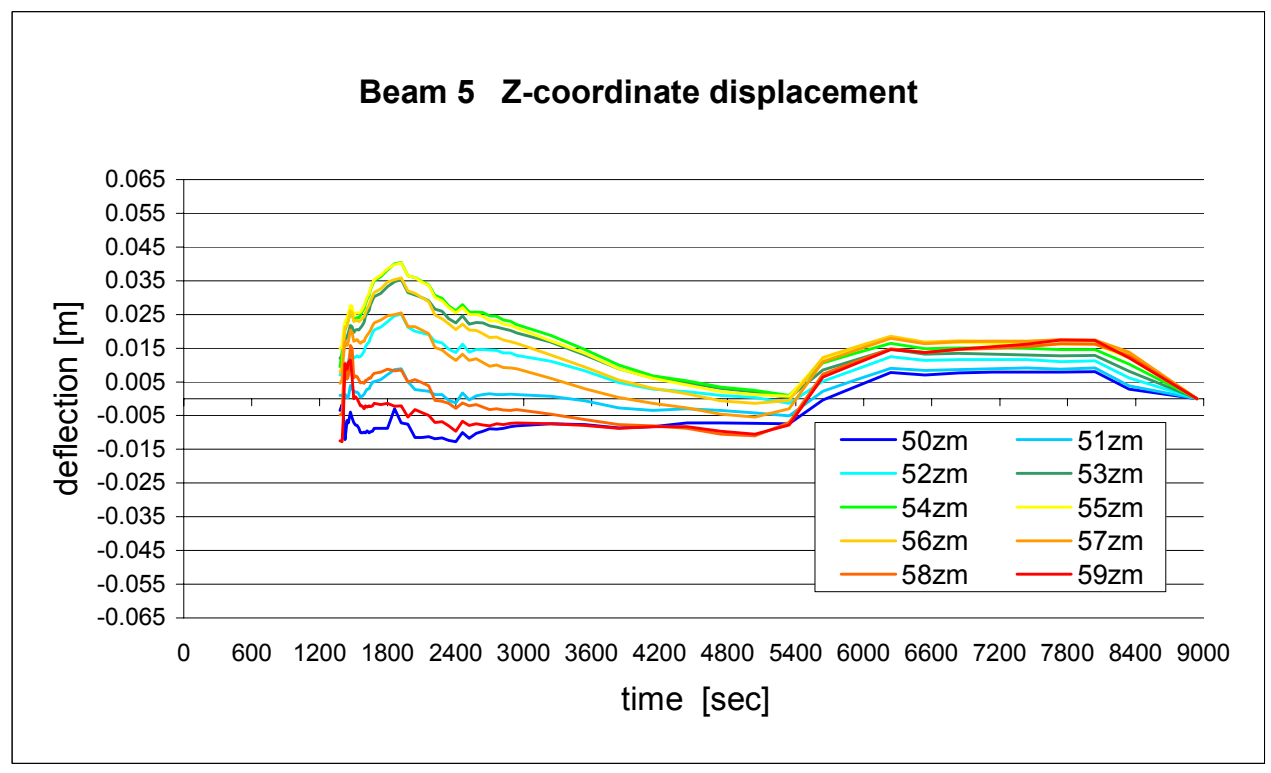

Fig. 7. Plots of vertical displacement versus time for the 10 targets on Beam 5. 
Application of the developed system to the monitoring of the super-hot steel beams produced relative accuracies in object space triangulation of 0.7-1.3 mm, which while being less than optimal were nevertheless sufficient for the determination of the desired deformation parameters.

\section{Acknowledgements}

This collaborative project owes much of its success to the participation and continual encouragement of Professor Wolfgang Niemeier and to the efforts of Dr. Ken Edmundson who performed the modifications to the Australis software.

\section{References}

Beyer, H.A., Uffenkamp, V., van der Vlugt, G., 1995. Quality Control in Industry with Digital Photogrammetry. In: Gruen/ Kahmen (Eds.), Optical 3-D Measurement Techniques III, Wichmann Verlag, Heidelberg, pp. 29-38.

Dold, J., 1996. Influence of large targets on the results of photogrammetric bundle adjustment. International Archives of Photogrammetry and Remote Sensing, Vol. 31, Part B5, pp. 119-123.

Fraser, C.S., 1983. Network Design Considerations for Non-Topographic Photogrammetry. Photogrammetric Engineering and Remote Sensing 50(8), 1115-1126.

Fraser, C.S., 1996. Industrial Measurement Applications, Chapter 12. In: Close Range Photo- grammetry and Machine Vision, K.B. Atkinson (Ed.), Whittles, Scotland, pp. 329-361.

Fraser, C.S. , 1999. Multi-Epoch Deformation Analysis via Vision Metrology. Proc. $3^{\text {rd }}$ Turkish-German Geodetic Days Conference "Towards A Digital Age", Istanbul, June 1-4, Vol. 1, pp. 49-58.

Fraser, C.S., Edmundson, K.L., 2000. Computational Processes in Digital Close-Range Photogrammetry. ISPRS Journal of Photogrammetry \& Remote Sensing 55(2), 94-104.

Niederöst, M., Maas, H.-G., 1997. Automatic Deformation Measurement with a Still Video Camera. In: Gruen/ Kahmen (Eds.), Optical 3-D Measurement Techniques IV, Wichmann Verlag, Heidelberg, pp. 266-271. 